Article

\title{
Structural Analysis of Gluco-Oligosaccharides Produced by Leuconostoc lactis and Their Prebiotic Effect
}

\author{
Sulhee Lee ${ }^{1,2} \mathbb{D}$, Jisun Park ${ }^{1}$, Jae-Kweon Jang ${ }^{3}$, Byung-Hoo Lee ${ }^{1}$ and Young-Seo Park ${ }^{1, *(D)}$ \\ 1 Department of Food Science and Biotechnology, Gachon University, Seongnam 13120, Korea; \\ sulhee2340@gmail.com (S.L.); jisunny4424@gmail.com (J.P.); blee@gachon.ac.kr (B.-H.L.) \\ 2 Research Group of Healthcare, Korea Food Research Institute, Wanju 55365, Korea \\ 3 Food Nutrition Major, School of Food, Chungkang College of Cultural Industries, Icheon 17390, Korea; \\ jkjang@chungkang.ac.kr \\ * Correspondence: ypark@gachon.ac.kr; Tel.: +82-31-750-5378
}

Received: 22 September 2019; Accepted: 5 November 2019; Published: 5 November 2019

\begin{abstract}
Leuconostoc lactis CCK940, which exhibits glycosyltransferase activity, produces oligosaccharides using sucrose and maltose as donor and receptor molecules, respectively. The oligosaccharides produced were purified by Bio-gel P2 chromatography and the purified oligosaccharides (CCK-oligosaccharides) consisted of only glucose. ${ }^{1} \mathrm{H}-\mathrm{NMR}$ analysis revealed that the CCK-oligosaccharides were composed of $77.6 \% \alpha-1,6$ and $22.4 \% \alpha-1,4$ glycosidic linkages, and the molecular weight of the CCK-oligosaccharides was found to be $9.42 \times 10^{2} \mathrm{Da}$. To determine the prebiotic effect of the CCK-oligosaccharides, various carbon sources were added in modified media. Growth of six probiotic strains, Lactobacillus casei, L. pentosus, L. plantarum, Weissella cibaria, Bifidobacterim animalis, and Saccharomyces cerevisiae, was better when the CCK-oligosaccharides were used as the sole carbon source compared to fructo-oligosaccharides, which are widely used as prebiotics. These results showed that the CCK-oligosaccharides produced from Leu. lactis CCK940 could serve as good candidates for novel prebiotics.
\end{abstract}

Keywords: prebiotics; probiotics; oligosaccharides; lactic acid bacteria; NMR

\section{Introduction}

Lactic acid bacteria produce carbohydrates, glycosides, oligosaccharides, or polysaccharides through glycosyltransferase activity [1,2]. Glycosyltransferase effectively synthesizes oligosaccharides such as galacto-oligosaccharides (GOS) and fructo-oligsoaccharides (FOS), which are widely used as prebiotics in industry [3-5]. The types of product synthesized by glycosyltransferase can be classified into dextransucrase (EC 2.4.1.5), alternansucrase (EC 2.4.1.140), mutansucrase (EC 2.4.1.5), and reuteransucrase (EC 2.4.1.5) [6,7]. The various oligosaccharides, which are synthesized by these enzymes, have linear chains which are composed of D-glucopyranosyl units. The dextran polymer has $\alpha-1,6$ glycosidic bonds, mutan polymer has $\alpha-1,3$ glycosidic bonds, alternan polymer consists of alternate $\alpha-1,6$ and $\alpha-1,3$ glycosidic bonds, and reuteran polymer has $\alpha-1,4$ glycosidic bonds $[7,8]$.

Prebiotics are typically defined as "selectively fermented, non-digestible food ingredients or substances that specifically support the growth and/or activity of health-promoting bacteria that colonize the gastrointestinal tract" [9]. This definition of prebiotics demonstrates that not all dietary carbohydrates are prebiotics, but they must satisfy the following criteria: (1) resistance to gastric acidity (low $\mathrm{pH}$ ), hydrolysis by mammalian enzymes, and gastrointestinal absorption; (2) fermentation by intestinal microflora; and (3) selective stimulation of the growth and/or activity 
of the intestinal bacteria that contribute to health and well-being [10,11]. Prebiotics typically contain FOS, GOS, isomalto-oligosaccharide (IMO), and cyclo-oligosaccharides. Many studies have shown that oligosaccharides as prebiotics exert positive effects on the human body. They improve intestinal function, stimulate the growth of beneficial bacteria, inhibit the growth of pathogens, increase short chain fatty acids (SCFAs), reduce total cholesterol and low-density lipoprotein (LDL) cholesterol, and enhance immune function [12-15].

In our previous reports, Leuconostoc lactis CCK940, which has high glucansucrase activity, was isolated from home-made kimchi (Korean traditional fermented vegetables) and the production of oligosaccharides from Leu. lactis CCK940 was optimized [14,16]. The purified oligosaccharides from Leu. lactis CCK940 showed immunostimulating effects on RAW264.7 macrophage cells [14].

Because oligosaccharides are well known prebiotics, it was predicted that the oligosaccharides produced by Leu. lactis CCK940 would also have prebiotic effects. Since only limited studies examining the prebiotic effects of oligosaccharides produced by lactic acid bacteria have been conducted, in the present study, we investigated the prebiotic effects of the CCK-oligosaccharides produced by Leu. lactis CCK940 using 24 probiotic strains.

\section{Results}

\subsection{Analysis of the Carbohydrate Composition of the CCK-Oligosaccharides}

To analyze the sugar composition, the CCK-oligosaccharides were acid-hydrolyzed by trifluoroacetic acid (TFA) and their hydrolyzed products were identified using thin-layer chromatography (TLC) and high-performance anion exchange chromatography with pulsed amperometric detection (HPAEC-PAD). As shown in Figure 1, the CCK-oligosaccharides were completely hydrolyzed by TFA; the HPAEC-PAD chromatograms of $0.005 \%(w / v)$ dextrose and hydrolysates were identical. The purified oligosaccharides were identified as gluco-oligosaccharides (CCK-oligosaccharides) consisting of only glucose.

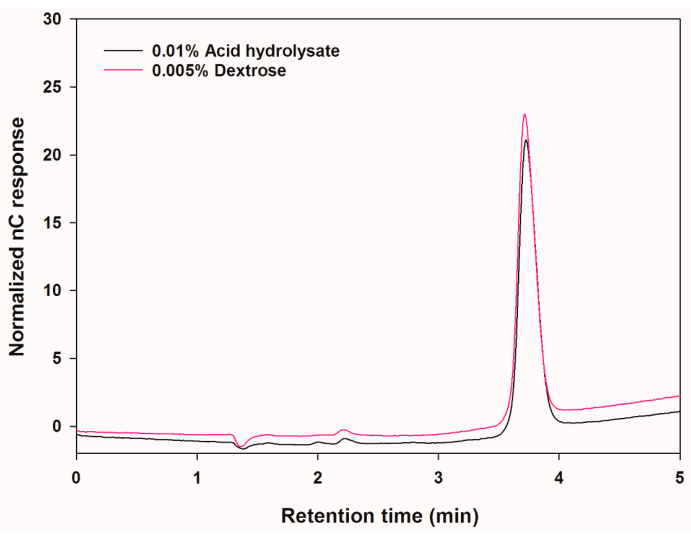

(a)

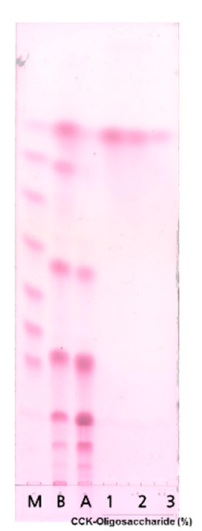

(b)

Figure 1. High-performance anion exchange chromatography with pulsed amperometric detection (HPAEC-PAD) and thin-layer chromatography (TLC) chromatograms of CCK-oligosaccharide (produced by L. lactis CCK940) acid hydrolysate. (a) HPAEC-PAD chromatogram of $0.005 \%(w / v)$ dextrose and $0.01 \%$ acid hydrolysate. (b) TLC chromatograms obtained before and after purification of CCK-oligosaccharides and acid-hydrolyzed CCK-oligosaccharides (M: glucose polymers G1-G7; B: before purification; A: after purification by Bio-gel P2; 1: $1 \%$ of acid hydrolysate; 2 : $0.5 \%$ of acid hydrolysate; $3: 0.2 \%$ of acid hydrolysate).

The molecular weight of the purified CCK-oligosaccharides was analyzed using size exclusion chromatography (Figure 2). Among the oligosaccharides that comprised the CCK-oligosaccharides, the molecular weight of the highest concentration oligosaccharide was determined to be $9.42 \times 10^{2} \mathrm{Da}$. 


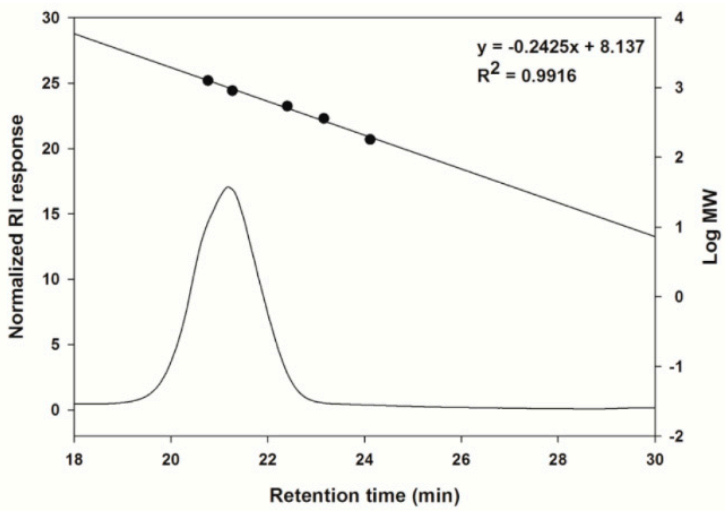

Figure 2. Size exclusion high-performance liquid chromatography (HPLC) chromatogram of the major CCK-oligosaccharides.

\subsection{Determination of the Types of Glycosidic Bonds}

To determine the types of glycosidic bonds in the CCK-oligosaccharides, the CCK-oligosaccharides were hydrolyzed with several carbohydrate hydrolysis enzymes and their products were analyzed by TLC (Figure 3). CCK-oligosaccharides were not hydrolyzed by $\alpha$-amylase, $\alpha$-glucosidase, $\beta$-glucosidase, lichenase, or $\beta$-1,3-D-glucanase. CCK-oligosaccharides were hydrolyzed by pullulanase M1 and produced oligosaccharides with a degree of polymerization (DP) of 11.

Figure 3. TLC chromatogram of enzymatic analysis of CCK-oligosaccharides. G: G1-G7 standard; C: CCK-oligosaccharides; aA: CCK-oligosaccharides treated with $\alpha$-amylase; aG: CCK-oligosaccharides treated with $\alpha$-glucosidase; bG: CCK-oligosaccharides with $\beta$-glucosidase; L: CCK-oligosaccharides treated with lichenase; b13: CCK-oligosaccharides treated with $\beta$-1,3-D-glucanase; Ag: CCK-oligosaccharides treated with amyloglucosidase; P: CCK-oligosaccharides treated with pullulanase M1; Ag+P: CCK-oligosaccharides treated with amyloglucosidase and pullulanase M1.

\subsection{Analysis of the Linkage Ratio by ${ }^{1} H-N M R$ Spectroscopy}

${ }^{1} \mathrm{H}-\mathrm{NMR}$ spectroscopy was used to analyze the linkage ratios of the CCK-oligosaccharides. Chemical shifts of CCK-oligosaccharides, maltose, nigerose, and waxy corn starch (WCS) were determined at 5-5.8 ppm (Figure 4). It was found that CCK-oligosaccharides were composed of only $\alpha$-linkages. WCS was used as a standard for determining the chemical shift of the resonances from the ${ }^{1} \mathrm{H}$ positions of $\alpha-1,4$ and $\alpha-1,6$ linkages. The results indicated that the percentages of the linkages for the CCK-oligosaccharides were 22.4\% $\alpha-1,6$ linkages and 77.6\% $\alpha-1,6$ linkages, and the branching ratio of $\alpha-1,4$ to $\alpha-1,6$ was calculated to be 0.3 (Table 1 ). 


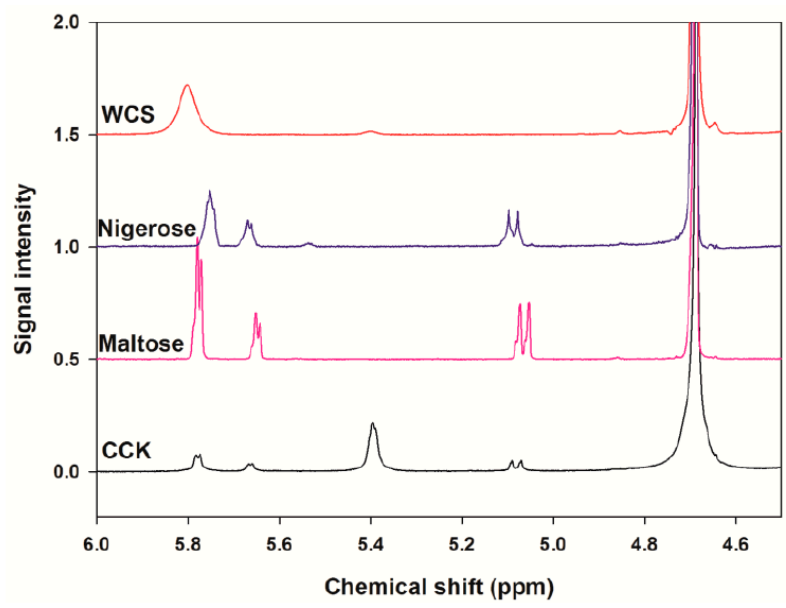

Figure 4. Comparison of the ${ }^{1} \mathrm{H}-\mathrm{NMR}$ spectra $\left(400 \mathrm{MHz}, \mathrm{D}_{2} \mathrm{O}\right)$ for CCK-oligosaccharides, maltose, nigerose, and waxy corn starch (WCS) (from the bottom).

Table 1. Relative abundance (\%) of $\alpha-1,4$ and $\alpha-1,6$ linkages in the CCK-oligosaccharides and WCS.

\begin{tabular}{cccc}
\hline & $\begin{array}{c}\boldsymbol{\alpha - 1 , 4} \\
\text { Linkages (\%) }\end{array}$ & $\begin{array}{c}\mathbf{1}-\mathbf{1 , 6} \\
\text { Linkages (\%) }\end{array}$ & $\begin{array}{c}\text { Ratio of } \\
\boldsymbol{\alpha - 1 , 4} \text { to } \boldsymbol{\alpha - 1 , 6}\end{array}$ \\
\hline WCS & $96.2 \pm 0.0$ & $3.8 \pm 0.0$ & $25.5 \pm 0.3$ \\
CCK-oligosaccharides & $22.4 \pm 1.2$ & $77.6 \pm 1.2$ & $0.3 \pm 0.0$ \\
\hline \multicolumn{2}{c}{ 1 Percentage was determined by calculating the area ratio from ${ }^{1} \mathrm{H}-\mathrm{NMR}}$.
\end{tabular}

\subsection{Prebiotic Effects of the CCK-Oligosaccharides}

To determine the prebiotic effects of the CCK-oligosaccharides, various carbon sources were added into modified media. Six of 24 strains, Lactobacillus casei, L. pentosus, L. plantarum, Weissella cibaria, Bifidobacterim animalis, and Saccharomyces cerevisiae were used to investigate CCK-oligosaccharides as prebiotics, compared to FOS which was used as a positive control (Figure 5). Viable cell numbers of L. casei, L. pentosus, and L. plantarum significantly increased in CCK media when compared with modified deMan, Rogosa, and Sharpe (MRS) (m-MRS) with FOS at 24 and $48 \mathrm{~h}$ of incubation. Viable cell numbers of $W$. cibaria were 9.36 and $4.35 \log$ CFU/mL in MRS at 12 and $48 \mathrm{~h}$, respectively. Viable cell numbers of $W$. cibaria were 8.01 and $7.94 \log$ CFU/mL in FOS and CCK media, respectively, at $48 \mathrm{~h}$ of incubation. B. animalis also exhibited results similar to $W$. cibaria; the viable cell number of $B$. animalis in the CCK medium was significantly higher than that in FOS media. B. animalis and S. cerevisiae grew better in media containing CCK-oligosaccharides than in FOS.

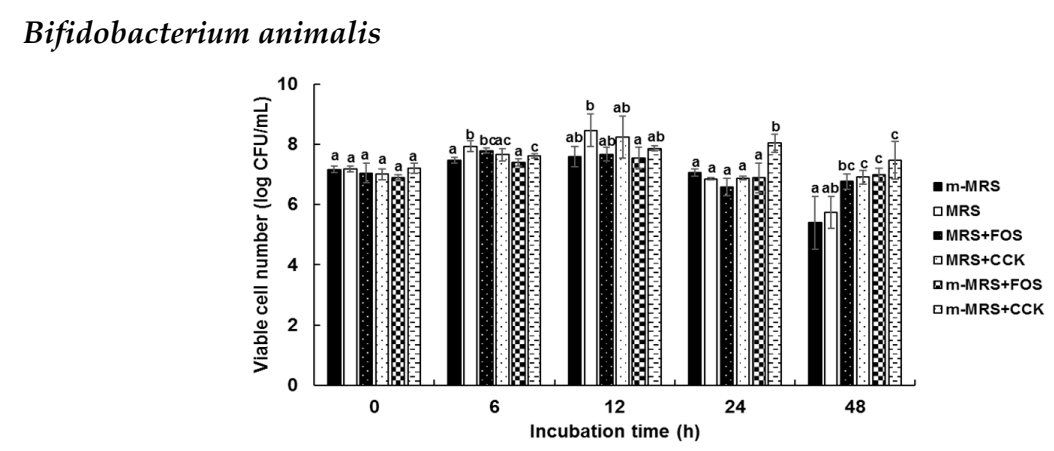

Figure 5. Cont. 
Lactobacillus casei

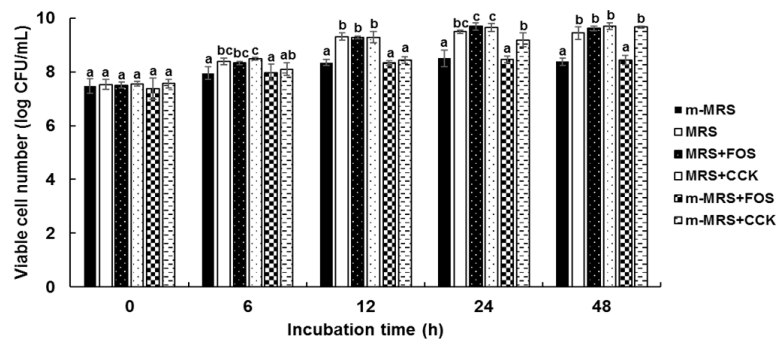

L. pentosus

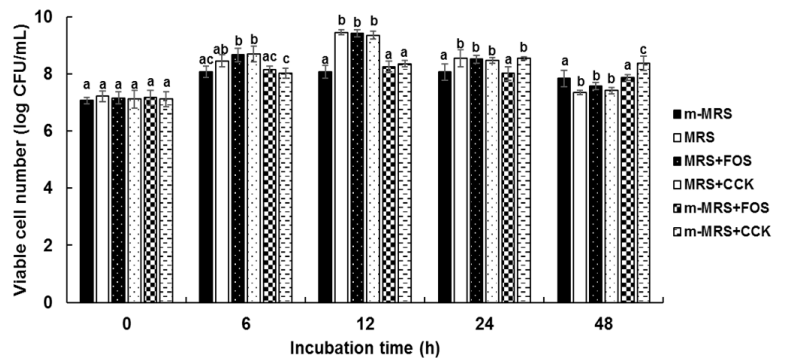

\section{L. plantarum}

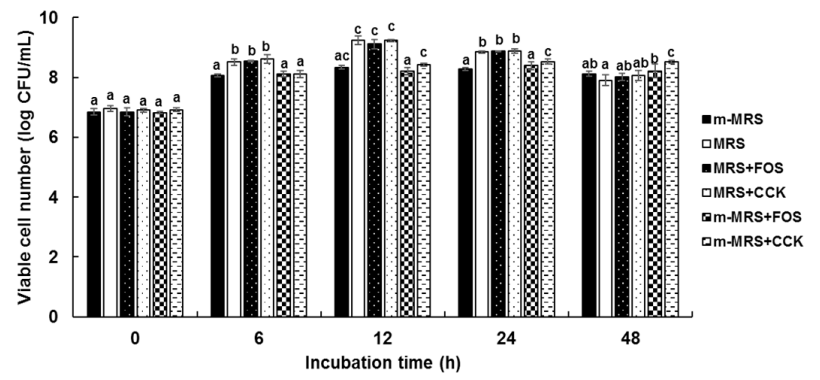

Weisella cibaria

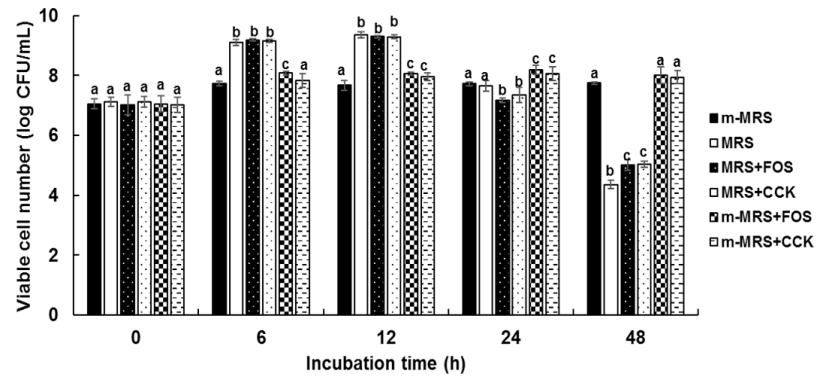

Saccharomyces cerevisiae

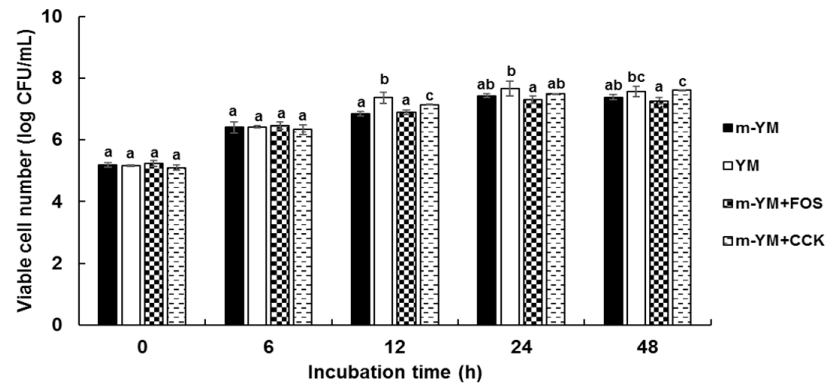

Figure 5. Prebiotic effects of CCK-oligosaccharides. One-way ANOVA was used for comparison of group mean values, followed by Duncan's multiple range test for significance of individual comparisons $(p<0.05)$. Different alphabet letters among groups represent statistically significant difference. 


\section{Discussion}

It has been reported that Leu. mesenteroides B-742 produces GOS and mannitol using maltose as an acceptor [17], and that Leu. mesenteroides NRRL B-23188 synthesizes gluco-oligosaccharides using calcium hydroxide-sucrose solution [3]. Although many studies on the synthesis of gluco-oligosaccharides by Leu. mesenteroides have been conducted, no studies have been conducted to investigate the gluco-oligosaccharides synthesized by Leu. lactis.

It has been reported that when pooled human milk oligosaccharides are fractionated, the molecular weight of the fractions is $2.094-3.626 \times 10^{3} \mathrm{Da}$ [18], and dextransucrase catalyzes the synthesis of oligosaccharides in the range of $50-100 \times 10^{3} \mathrm{Da}$ [19]. The molecular weight of CCK-oligosaccharides was lower than that of other oligosaccharides.

$\alpha$-Amylase (EC 3.2.1.1) hydrolyzes the $\alpha-1,4$ glucose linkages of starch through an endo-type mechanism and $\alpha$-glucosidase (EC 3.2.1.20) cleaves a glucose unit from the non-reducing end of $\alpha-1,4$ oligosaccharides and polysaccharide starch fragments through an exo-type mechanism. CCK-oligosaccharides were not hydrolyzed by $\alpha$-amylase or $\alpha$-glucosidase, which implies that these carbohydrates are non-digestible in the human intestinal tract because $\alpha$-amylase and $\alpha$-glucosidase are major carbohydrate-hydrolyzing enzymes in the human digestive system. $\beta$-Glucosidase (EC 3.2.1.21) releases a glucose unit from the non-reducing end of $\beta-1,4$ or $\beta-1,3$ glycosidic bonds of oligosaccharides or polysaccharide fragments, lichenase hydrolyzes $\beta-1,4$ glycosidic linkages in $\beta$-glucans containing $\beta-1,3$ and $\beta-1,4$ bonds, and $\beta-1,3-\mathrm{D}-$ glucanase (EC 3.2.1.6) cleaves $\beta-1,3$ linkages. CCK-oligosaccharides were not hydrolyzed by these three enzymes. Amyloglucosidase (EC 3.2.1.3) hydrolyzes $\alpha-1,4$ and $\alpha-1,6$ glycosidic bonds from the non-reducing end of starch oligomers and polymers through an exo-type mechanism, and this enzyme hydrolyzed CCK-oligosaccharides and released glucose. Pullulanase M1 is an endo-type enzyme similar to amyloglucosidase, which hydrolyzes $\alpha-1,6$ bonds of pullulan, amylopectin, glycogen, and $\alpha$ - and $\beta$-limit dextrins of amylopectin and glycogen. CCK-oligosaccharides were hydrolyzed by pullulanase M1 and produced oligosaccharides with a degree of polymerization (DP) of 11. Based on these results, it was predicted that there are no $\beta-1,3$ or $\beta-1,4$ glycosidic linkages in the CCK-oligosaccharides.

Nuclear magnetic resonance (NMR) is the most powerful tool available for evaluating oligosaccharide linkage ratios [20]. Generally, the chemical shift of an anomeric proton was detected at 4.3-5.9 ppm in the ${ }^{1} \mathrm{H}-\mathrm{NMR}$ spectra. Maltose and nigerose are disaccharides formed from two units of glucose joined with an $\alpha-1,4$ bond and connected with an $\alpha-1,3$ linkage, respectively. Waxy corn starch (WCS) consists of polysaccharides formed from glucose polymers joined with $\alpha-1,4$ and $\alpha-1,6$ bonds. The enzyme 4,6- $\alpha$-glucanotransferase of L. reuteri 121 acts on malto-oligosaccharides (MOS); the MOS consist of $\alpha-1,4$ and $\alpha-1,6$ glycosidic linkages [21]. Dextran and alternan were produced by Leu. mesenteroides NRRL B-512F; dextran contains approximately $95 \% \alpha-1,6$ linkages; however, alternan contains alternating $\alpha-1,3$ and $\alpha-1,6$ linkages [22]. The structure of CCK-oligosaccharides was different from other oligosaccharide in that branching ratio of $\alpha-1,4$ to $\alpha-1,6$ was lower than others.

When the prebiotic effect of a sugar is examined, minimal medium without a carbon source can be used so that the supplemented sugar is the sole carbon source. However, because lactic acid bacteria are fastidious bacteria, there is no suitable minimal medium for the growth of lactic acid bacteria. MRS medium, which is usually used for the cultivation of lactic acid bacteria, is a complex medium, but the beef extract and yeast extract in MRS are utilized as nitrogen sources rather than carbon sources by lactic acid bacteria [23]. Therefore, use of MRS medium without glucose is alternative for the examination of prebiotic effects. Furthermore, because the intestinal environment track is rich in a variety of nutrients, the use of a complex medium such as MRS medium rather than a minimal medium is a good way to understand the prebiotic activity of oligosaccharides. For the same reason, Yeast Mold (YM) without glucose was used in this study for the growth of yeast instead of using yeast nitrogen base, the minimal medium generally used for yeast [24].

$W$. cibaria showed the highest viable cell number at $12 \mathrm{~h}$ of incubation, following which the viable cell number decreased. This phenomenon was attributed to the production of acid. It has been reported 
that gentio-oilgosaccharide, which is synthesized by Leu. mesenteroides NRRL B-1426, exerts a prebiotic effect on B. infantis and L. acidophilus [25] and $\alpha-(1,6)$ - and $\alpha-(1,3)$-linked oligosaccharides with DP 3 , which are synthesized by alternansucrase of Leu. mesenteroides NRRL B-21297, exhibit a good prebiotic effect [26].

Growth of the six probiotic strains, Lactobacillus casei, L. pentosus, L. plantarum, Weissella cibaria, Bifidobacterim animalis, and Saccharomyces cerevisiae, was better when the CCK-oligosaccharides were used as the sole carbon source compared to fructo-oligosaccharides, which are widely used as prebiotics.

\section{Materials and Methods}

\subsection{Bacterial Strains and Culture Conditions}

The strain Leu. lactis CCK940 (GenBank accession number NZ_NQLF00000000) used in this study was isolated from home-made kimchi [16]. This strain was cultured using Lactobacillus MRS broth (BD, Franklin Lakes, NJ, USA) at $30^{\circ} \mathrm{C}$ for $20 \mathrm{~h}$ as a seed culture.

\subsection{Determination of Oligosaccharide Structure}

\subsubsection{Purification of Oligosaccharides}

The oligosaccharides from Leu. lactis CCK940 were produced in optimized fermentation conditions and purified as described in a previous report [14].

\subsubsection{Thin-Layer Chromatography (TLC)}

Samples were spotted onto silica gel 60 F254 (Merck, Darmtadt, Germany), and the gel plates were developed twice using 2:5:1.5 nitromethane (Sigma-Aldrich, St. Louis, MO, USA):n-propyl alcohol (Samchun, Gyeonggi, Korea):water. The developed TLC plate was dipped in 0.3\% (w/v) N-(1-naphtyl) ethylenediamine dihydrochloride (Sigma-Aldrich) and 5\% (v/v) sulfuric acid (Duksan, Seoul, Korea) in methanol (CARLO ERBA Reagents S.A.A., Val de Reuil, France), and then baked at $121^{\circ} \mathrm{C}$ for $5 \mathrm{~min}$. Glucose polymers (G1-G7, of which the DPs are 1-7, respectively) purchased from Carbosynth Co. (Berkshire, UK) were used as standard sugars.

\subsubsection{HPAEC-PAD Analysis}

The CCK-oligosaccharides produced by Leu. lactis CCK940 were analyzed using a HPAEC-PAD (DX 500 Chromatography System, Dionex, Sunnyvale, CA, USA) [14]). The Leu. lactis CCK940 culture was centrifuged at $27,237 \times g$ for $1 \mathrm{~min}$ and the supernatant was analyzed by HPAEC-PAD (Dionex) with a CarboPac PA-1 column $(4 \times 250 \mathrm{~mm}$, Dionex $)$ and a CarboPac PA-1 guard column $(4 \times 50 \mathrm{~mm}$, Dionex). The flow rate was $1.0 \mathrm{~mL} / \mathrm{min}$, and the mobile phase used for oligosaccharide separation was $150 \mathrm{mM}$ sodium hydroxide for the first $20 \mathrm{~min}$; subsequently, $600 \mathrm{mM}$ sodium acetate (in 150 $\mathrm{mM}$ sodium hydroxide) was applied with a gradient of 60:40 to 0:100 from 20 to $25 \mathrm{~min}$, and $150 \mathrm{mM}$ sodium hydroxide was used from 25 to 40 min (sodium hydroxide, Fisher Scientific, Hampton, NH, USA; sodium acetate, Sigma-Aldrich). Ten microliters of each sample was injected for each analysis.

\subsubsection{Size Exclusion HPLC Analysis}

The size of the CCK-oligosaccharides produced by Leu. lactis CCK940 was analyzed using size exclusion chromatography. The CCK-oligosaccharides $(0.1 \%, w / v)$ were dissolved in distilled water and the soluble CCK-oligosaccharides were analyzed by HPLC (UltiMate ${ }^{\mathrm{TM}} 3000$ RSLC nano system, Thermo Fisher scientific, Inc., Waltham, MA, USA) equipped with an OHpak SB-802.5 column $(8.0 \times$ $300 \mathrm{~mm}$, Shodex, New York, NY, USA). The flow rate was $0.4 \mathrm{~mL} / \mathrm{min}$, column oven temperature was $35^{\circ} \mathrm{C}$, the mobile phase used for CCK-oligosaccharides separation was filtered and distilled water, and they were detected by RI (RI-101, Shodex). One hundred microliters of sample was injected for 
analysis. Glucose polymers (DP 1-7) purchased from Carbosynth Co. (Berkshire, UK) were used as a standard sugar.

\subsubsection{Analysis of Sugar Composition}

The CCK-oligosaccharides were hydrolyzed with $4 \mathrm{M}$ TFA at $121^{\circ} \mathrm{C}$ for $2 \mathrm{~h}$. TFA was then removed by evaporation under gentle $\mathrm{N}_{2}$ gas flow. The sugar components of the CCK-oligosaccharides were analyzed by HPAEC-PAD and TLC.

\subsubsection{Composition of Glycosidic Bonds by Enzymatic Analysis}

CCK-oligosaccharides were treated with various carbohydrate-hydrolyzing enzymes to determine the composition of the glycosidic bonds. CCK-oligosaccharides $(1 \%, w / v)$ were treated with the following enzymes and concentrations: $10 \mathrm{mU}$ of $\alpha$-amylase, $100 \mathrm{mU}$ of $\alpha$-glucosidase, $520 \mathrm{mU}$ of amyloglucosidase (Sigma-Aldrich), $1.4 \mathrm{U}$ of pullulanase M1, $10 \mathrm{mU}$ of lichenase (Megazyme, Chicago, IL, USA), $100 \mathrm{mU}$ of $\beta$-glucosidase, and $10 \mathrm{mU}$ of $\beta$-1,3-D-glucanase (Sigma-Aldrich). The CCK-oligosaccharides were reacted with enzymes at $37^{\circ} \mathrm{C}$ for $1 \mathrm{~h}$ and the products were determined by TLC.

\subsubsection{Analysis of Linkage Ratio by Proton Nuclear Magnetic Resonance $\left({ }^{1} \mathrm{H}-\mathrm{NMR}\right)$ Spectroscopy}

The relative abundance of $\alpha-1,4$ and $\alpha-1,6$ linkages in the CCK-oligosaccharides was determined by $400 \mathrm{MHz}{ }^{1} \mathrm{H}-\mathrm{NMR}$ spectroscopy (JeolJNM-LA400 with LFG, JEOL, Tokyo, Japan) and Delta NMR Processing and Control Software version 5.3 (JEOL USA, Inc., Peabody, MA, USA). Freeze-dried samples $(10 \mathrm{mg} / \mathrm{mL})$ were first dissolved in deuterium oxide $\left(\mathrm{D}_{2} \mathrm{O}\right)$ and then freeze-dried again. Samples dissolved in $\mathrm{D}_{2} \mathrm{O}(10 \mathrm{mg} / \mathrm{mL})$ were analyzed by ${ }^{1} \mathrm{H}-\mathrm{NMR} .{ }^{1} \mathrm{H}-\mathrm{NMR}$ spectra were collected at $80^{\circ} \mathrm{C}$.

\subsection{Prebiotic Effects}

\subsubsection{Selection of Probiotic Strains}

A total of 24 probiotic strains were obtained from the Korean Culture Center of Microorganisms (KCCM, Seoul, Korea); B. adolescentis KCCM 11206, B. animalis KCCM 11209, B. bifidum KCCM 12096, B. breve KCCM 42255, B. longum KCCM 11953, Enterococcus faecium KCCM 12118, L. acidophilus KCCM 32820, L. casei KCCM 12452, L. fermentum KCCM 35469, L. helveticus KCCM 40989, L. johnsonii KCCM 41274, L. paracasei KCCM 40995, L. pentosus KCCM 40997, L. plantarum KCCM 12116, L. rhamnosus KCCM 32405, L. salivarius KCCM 40210, Lactococcus lactis KCCM 40104, Leuconostoc citreum KCCM 12030, Leu. mesenteroides KCCM 11325, Pediococcus pentosaceus KCCM 11902, Streptococcus thermophilus KCCM 35496, and W. cibaria KCCM 41287. These strains were cultured in MRS medium in the presence or absence of prebiotics at $37^{\circ} \mathrm{C}$ for $48 \mathrm{~h}$. S. cerevisiae KCCM 50549 and Zygosaccharomyces rouxii KCCM 12066 were cultured using YM medium at $30^{\circ} \mathrm{C}$.

\subsubsection{Growth Conditions of Probiotic Strains}

To determine the prebiotic effects of the CCK-oligosaccharides produced by Leu. lactis CCK940, selected probiotic strains were incubated with medium containing CCK-oligosaccharides as the sole carbon source. m-MRS and modified YM (m-YM) had no carbon sources (Tables 2 and 3). For lactic acid bacteria, m-MRS was used as a negative control, commercial Lactobacillus MRS (MRS containing $2 \%(w / v)$ dextrose) was used as a positive control, MRS with $1 \%(w / v)$ FOS, MRS with $1 \%(w / v)$ CCK-oligosaccharides (CCK), m-MRS with $1 \%(w / v)$ FOS, and m-MRS with $1 \%(w / v)$ CCK media were used. For yeast strains, $\mathrm{m}-\mathrm{YM}$ was used as a negative control, commercial $\mathrm{YM}$ (YM containing 1\% $(w / v)$ dextrose) was used as a positive control, and m-YM with $1 \%(w / v)$ FOS, and m-YM with $1 \%(w / v)$ CCK media were used. 
Table 2. Components of the modified MRS media.

\begin{tabular}{cc}
\hline Ingredient & Amount $(\mathrm{g} / \mathrm{L})$ \\
\hline Proteose peptone No. 3 & 10.0 \\
Beef extract & 10.0 \\
Yeast extract & 5.0 \\
Polysorbate 80 & 1.0 \\
Ammonium citrate & 2.0 \\
Sodium acetate & 5.0 \\
Magnesium sulfate & 0.1 \\
Manganese sulfate & 0.05 \\
Dipotassium phosphate & 2.0 \\
\hline
\end{tabular}

Table 3. Components of the modified YM media.

\begin{tabular}{cc}
\hline Ingredient & Amount $(\mathrm{g} / \mathrm{L})$ \\
\hline Yeast extract & 3.0 \\
Malt extract & 3.0 \\
Peptone & 5.0 \\
\hline
\end{tabular}

\subsubsection{Microbiological Analysis}

A colony of lactic acid bacteria was seeded in MRS broth and incubated at $37{ }^{\circ} \mathrm{C}$ for $24 \mathrm{~h}$. Additionally, one colony of yeast was seeded in YM broth and incubated at $30^{\circ} \mathrm{C}$ for $24 \mathrm{~h}$ at $250 \mathrm{rpm}$. One percent $(v / v)$ culture broth was inoculated in various media. Bifidobacterium sp., lactic acid bacteria, and yeast were incubated in anaerobic, facultative anaerobic, and aerobic conditions, respectively. Culture broth was sampled at $0,12,24$, and $48 \mathrm{~h}$. Bifidobacterium sp. and lactic acid bacteria were spread on MRS agar plates and yeast was spread on YM agar plates.

\subsection{Statistical Analysis}

Data are presented as mean \pm standard deviation (SD) from triplicate experiments. Statistical analyses were performed using SPSS 23 (SPSS Inc., Chicago, IL, USA). Statistical significance between groups was determined by one-way analysis of variance (ANOVA), followed by Duncan's multiple range test $(p<0.05)$.

\section{Conclusions}

To the best of our knowledge, this is the first study to investigate the gluco-oligosaccharides produced from Leu. lactis strains, although several studies have been conducted to examine the gluco-oligosaccharides produced by other lactic acid bacteria. The CCK-oligosaccharides found were novel oligosaccharides based on their structure, and we found that the glycosidic linkages were composed of $77.6 \% \alpha-1,6$ and $22.4 \% \alpha-1,4$. The CCK-oligosaccharides significantly promoted the growth of L. casei, L. pentosus, L. plantarum, W. cibaria, B. animalis, and S. cerevisiae, the major probiotic strains used in the food industry. The prebiotic activity of the CCK-oligosaccharides was better than FOS, which is a major commercially available prebiotic, in some probiotic strains. The prebiotic activities of CCK-oligosaccharides and FOS were found to be strain-dependent. This showed the potential suitability of the use of CCK-oligosaccharides as prebiotics and their significance in the rapidly growing market for prebiotics.

Author Contributions: Y.-S.P. and J.-K.J. conceived and designed the experiments and proofread the manuscript; S.L. performed the experiments, analyzed the data and wrote the paper; J.P. performed the experiments for prebiotics effect; B.-H.L. performed the experiments for structural analysis of oligosaccharides.

Funding: This research was funded by the Korea government (Ministry of Science and ICT), grant number (2019R1A2C1004950). 
Acknowledgments: This work was supported by the National Research Foundation of Korea (NRF) grant funded by the Korea government (MSIT) (No. 2019R1A2C1004950).

Conflicts of Interest: The authors declare no conflicts of interest.

\section{References}

1. Coutinho, P.M.; Deleury, E.; Davies, G.J.; Henrissat, B. An evolving hierarchical family classification for glycosyltransferase. J. Mol. Biol. 2003, 328, 307-317. [CrossRef]

2. Lairson, L.L.; Henrissat, B.; Davies, G.J.; Withers, S.G. Glycosyltransferase: Structures, functions, and mechanisms. Ann. Rev. Biochem. 2008, 77, 521-555. [CrossRef] [PubMed]

3. Lee, S.; Hanh, N.T.T.; Cho, J.Y.; Kim, J.Y.; Moon, Y.H.; Yeom, S.C.; Kim, G.J.; Kim, D. Glucooligosaccharide production by Leuconostoc mesenteroides fermentation with efficient $\mathrm{pH}$ control, using a calcium hydroxide-sucrose solution. Biotechnol. Bioprocess Eng. 2016, 21, 39-45. [CrossRef]

4. Monsan, P.F.; Ouarné, F. Chapter 10. Oligosaccharides derived from sucrose. In Prebiotics and Probiotics Science and Technology; Charalampopoulos, D., Rastall, R.A., Eds.; Springer Science+Business Media: New York, NY, USA, 2009; pp. 293-336.

5. Naessens, M.; Cerdobbel, A.; Soetaert, W.; Vandamme, E.J. Leuconostoc dextransucrase and dextran: Production, properties and applications. J. Chem. Technol. Biotechnol. 2005, 80, 845-860. [CrossRef]

6. Heincke, K.; Demuth, B.; Jördening, H.J.; Buchholz, K. Kinetics of the dextransucrase acceptor reaction with maltose-experimental results and modeling. Enzyme Microb. Technol. 1999, 24, 523-534. [CrossRef]

7. Leemhuis, H.; Pijning, T.; Dobruchowska, J.M.; van Leeuwen, S.S.; Kralj, S.; Dijkstra, B.W.; Dijkhuizen, L. Glucansucrases: Three-dimensional structures, reactions, mechanism, $\alpha$-glucan analysis and their implications in biotechnology and food applications. J. Biotechnol. 2013, 163, 250-272. [CrossRef]

8. Monchois, V.; Willemot, R. -M.; Monsan, P. Glucansucrases: Mechanism of action and structure function relationships. FEMS Microbiol. Rev. 1999, 23, 131-151. [CrossRef]

9. Bindels, L.B.; Delzenne, N.M.; Cani, P.D.; Walter, J. Towards a more comprehensive concept for prebiotics. Nat. Rev. Gastroenterol. Hepatol. 2015, 12, 303-310. [CrossRef]

10. de Vrese, M.; Schrezenmeir, J. Probiotics, prebiotics, and synbiotics. Adv. Biochem. Eng. Biotechnol. 2008, 111, 1-66. [CrossRef]

11. Roberfroid, M. Prebiotics: The concept revisited. J. Nutr. 2007, 137, 830-837. [CrossRef]

12. Costabile, A.; Fava, F.; Röytiö, H.; Forssten, S.D.; Olli, K.; Klievink, J.; Rowland, I.R.; Ouwehand, A.C.; Rastall, R.A.; Gibson, G.R.; et al. Impact of polydextrose on the faecal microbiota: A double-blind, crossover, placebo-controlled feeding study in healthy human subjects. Br. J. Nutr. 2012, 108, 471-481. [CrossRef] [PubMed]

13. Putaala, H.; Mäkivuokko, H.; Tiihonen, K.; Rautonen, N. Simulated colon fiber metabolome regulates genes involved in cell cycle, apoptosis, and energy metabolism in human colon cancer cells. Mol. Cell. Biochem. 2011, 357, 235-245. [CrossRef] [PubMed]

14. Lee, S.; Park, G.-G.; Jang, J.-K.; Park, Y.-S. Optimization of oligosaccharide production from Leuconostoc lactis using a response surface methodology and the immunostimulating effects of these oligosaccharides on macrophage cells. Molecules 2018, 23, 2118. [CrossRef] [PubMed]

15. Chung, C.H. Production of glucooligosaccharides and mannitol from Leuconostoc mesenteroides B-742 fermentation and its separation from byproducts. J. Microbiol. Biotechnol. 2006, 16, 325-329.

16. Finke, B.; Stahl, B.; Pfenninger, A.; Karas, M.; Daniel, H.; Sawatzki, G. Analysis of high-molecular-weight oligosaccharides from human milk by liquid chromatography and MALDI-MS. Anal. Chem. 1999, 71, 3755-3762. [CrossRef] [PubMed]

17. Koepsell, H.J.; Tsuchiya, H.M.; Hellman, N.N.; Kazenko, A.; Hoffman, C.A.; Sharpe, E.S.; Jackson, R.W. Enzymatic synthesis of dextran, acceptor specificity and chain initiation. J. Biol. Chem. 1953, 200, 793-801.

18. Gidley, M.J. Quantification of the structural features of starch polysaccharides by NMR spectroscopy. Carbohydr. Res. 1985, 139, 85-93. [CrossRef]

19. Dobruchowska, J.M.; Gerwig, G.J.; Kralj, S.; Grijpstra, P.; Leemhuis, H.; Dijkhuizen, L.; Kamerling, J.P. Structural characterization of linear isomalto-/malto-oligomer products synthesized by the novel GTFB 4,6- $\alpha$-glucanostransferase enzyme from Lactobacillus reuteri 121. Glycobiology 2012, 22, 517-528. [CrossRef] 
20. Côté, G.L. Alternan. In Biopolymers; Steinbüchel, A., Ed.; Wiley-VCH: Weinheim, Germany, 2002; Volume 5, pp. 323-350.

21. Kothari, D.; Goyal, A. Gentio-oligosaccharides from Leuconostoc mesenteroides NRRL B-1426 dextransucrase as prebiotics and as a supplement for functional foods with anti-cancer properties. Food Funct. 2015, 6, 604-611. [CrossRef] [PubMed]

22. Sanz, M.; Côté, G.; Gibson, G.; Rastall, R. Prebiotic properties of alternansucrase maltose-acceptor oligosaccharides. J. Agric. Food Chem. 2005, 53, 5911-5916. [CrossRef]

23. Hayek, S.A.; Ibrahim, S.A. Current limitations and challenges with lactic acid bacteria: A review. Food Nutr. Sci. 2013, 73-87. [CrossRef]

24. Abelovska, L.; Bujdos, M.; Kubova, J.; Petrezselyove, S.; Nosek, J.; Tomaska, L. Comparison of element levels in minimal and complex yeast media. Can. J. Microbiol. 2007, 53, 533-535. [CrossRef] [PubMed]

25. Lee, S.; Park, Y.-S. Oligosaccharide production by Leuconostoc lactis CCK940 which has glucansucrase activity. Food Eng. Prog. 2017, 21, 383-390. [CrossRef]

26. Astolfi, M.L.; Protano, C.; Schiavi, E.; Marconi, E.; Capobianco, D.; Massimi, L.; Ristorini, M.; Baldassarre, M.E.; Laforgia, N.; Vitali, M.; et al. A prophylactic multi-strain probiotic treatment to reduce the absorption of toxic elements: In-vitro study and biomonitoring of breast milk and infant stools. Environ. Int. 2019, 130, 104818. [CrossRef] [PubMed]

Sample Availability: Not available. 